\title{
The effects of a TNF-alpha inhibitor and HBO combination on the severity of pancreatitis and oxidative response in an experimental model of acute pancreatitis
}

\author{
Tasci $\mathrm{S}^{1}$, Senocak R², Lapsekili E², Kaymak $\mathrm{S}^{2}$, Yigit $\mathrm{T}^{3}$ \\ Department of General Surgery, Imamoglu State Hospital, Adana, Turkey. sahinkaymak@hotmail.com
}

\begin{abstract}
OBJECTIVES: We aimed to investigate the effects of infliximab and HBO (hyperbaric oxygen) used alone or in combination on oxidative stress and the severity of pancreatitis in an experimental model of AP (acute pancreatitis). MATERIAL AND METHODS: A total of 60 rats were randomly divided into five groups. Group 1 underwent laparotomy; Group 2 underwent experimental AP; Group 3 was given an infliximab infusion and underwent AP; Group 4 was subjected to HBO therapy after AP; and Group 5 was given infliximab infusion before AP and subjected to HBO therapy. Serum amylase, superoxide dismutase (SOD), malondialdehyde (MDA), and glutathione peroxidase (GPX) levels in the pancreas tissues were measured. The pancreatic tissue samples were scored. RESULTS: There were statistically significant differences in the histopathological scores and amylase levels between non-treated AP and all the three treatment groups. Group 5 had the closest histopathological scores to the sham group. MDA levels were significantly different between non-treated AP and all the three treatment groups, but the SOD levels and GPX values were not.

CONCLUSIONS: Combination of HBO therapy and Infliximab showed a synergistic effect on the reduction of histopathological severity and mortality in acute pancreatitis. All treatment modalities reduced the pathological findings by decreasing lipid peroxidation and partly increasing the antioxidant capacity in early period (Tab. 1, Fig. 3, Ref. 28). Text in PDF www.elis.sk.

KEY WORDS: acute pancreatitis, hyperbaric oxygen preconditioning, oxidative stress, TNF- $\alpha$ inhibitors.
\end{abstract}

\section{Introduction}

Acute pancreatitis (AP) is a disease, which progresses with acinar cell damage, and presents with pathological findings that vary from edema to necrosis. The mortality of AP ranges between $10-30 \%$, and the disease is still a major concern due to its clinically relevant consequences (1). Although AP may have different etiologies, the clinical presentation typically includes auto-degeneration of the tissue upon the activation of pancreatic enzymes and the consequent inflammation caused by the release of such key pro-inflammatory cytokines as interleukins (ILs) and tumor necrosis factor-alpha (TNF- $\alpha)(2)$. TNF- $\alpha$ promotes inflammatory cells infiltration including neutrophils and macrophages to accumulate in the pancreas, which results in exacerbating the local inflammatory response in the pancreas and progressing into a systemic inflammatory response (3).

${ }^{1}$ Department of General Surgery, Imamoglu State Hospital, Adana, Turkey, ${ }^{2}$ Department of General Surgery, Gulhane School of Medicine, Ankara, and ${ }^{3}$ Department of Metabolic sSurgery, Etiler Special Hospital, Istanbul, Turkey

Address for correspondence: S. Kaymak, Department of General Surgery, Gulhane School of Medicine, 06018, Etlik, Ankara, Turkey.

Phone: +90312.3045112, Fax: +90312.3045002
Infliximab is a chimeric anti-TNF- $\alpha$ monoclonal antibody. By binding both the water-soluble and transmembrane forms of TNF- $\alpha$, it induces the destruction of TNF- $\alpha$-producing cells and inhibits the activity of this cytokine through the complement fixation. There is a limited number of experimental studies investigating the efficacy of infliximab in the treatment of $\mathrm{AP}(4,5)$.

However, the effects of hyperbaric oxygen (HBO) therapy on AP have been investigated in a number of experimental models of AP, which showed decreased oxidative stress parameters and histopathological scores with increased levels of antioxidant products $(6,7)$. There is yet no specific drug to neutralize the inflammatory process of pancreatitis and no single agent alone to repair the damage. Thus, in the present study, we aimed to investigate the effects of infliximab, a TNF- $\alpha$ inhibitor, and HBO, used alone or in combination, on oxidative stress and the severity of pancreatitis in an experimental model of AP.

\section{Material and methods}

After approval was obtained from the institutional Ethics Committee, the study was carried out at Gulhane, School of Medicine, Research \& Development Central Animal Laboratories, Ankara, Turkey between 2009 and 2010. A total of 60 female Wistar rats weighing between 280 and $350 \mathrm{~g}$ were used in the study. All ani- 
mals were kept for one week in fixed-temperature cages in a 12-h daylight/nighttime cycle, and all were fed with standard rat feed and water. For $12 \mathrm{~h}$ prior to the experiment, the rats were given only water.

\section{Study protocol}

A total of 60 rats were randomly assigned to one of the five groups. Group 1 (sham, $\mathrm{n}=12$ ) underwent laparotomy and pancreas manipulation; Group 2 (non-treated AP, $n=12$ ) underwent laparotomy and experimental AP; Group 3 (infliximab + AP, $n$ $=12$ ) was given infliximab through a tail vein using an infusion pump for $20 \mathrm{~min}$ at a dose of $8 \mathrm{mg} / \mathrm{kg}$ (Remicade ${ }^{\circledR}$, ScheringPlough, Istanbul, Turkey), immediately prior to laparotomy and underwent AP induction; Group $4(\mathrm{Ap}+\mathrm{HBO} ; \mathrm{n}=12)$ was given HBO therapy for $48 \mathrm{~h}$ under 2.5 ATA pressure at a total of four sessions, given as 90 min sessions every $12 \mathrm{~h}$, beginning in the postoperative sixth hour after AP; and Group 5 (infliximab + AP $+\mathrm{HBO}, \mathrm{n}=12$ ) was given infliximab per protocol immediately prior to AP induction and underwent HBO therapy per protocol. All rats were sacrificed at $48 \mathrm{~h}$ following the experiment through the intracardiac blood withdrawal method.

\section{AP Model}

Acute pancreatitis was induced in the study through the intraductal retrograde bile acid infusion method, which was previously been shown to resemble human pancreatitis. The abdomen was accessed through a 3-cm midline incision performed under ketamine-HCI (Ketalar Flk ${ }^{\circledR}$, Pfizer) anesthesia. The duodenum wall was punctured by a 28-gauge catheter with an inserted $1 \mathrm{cc}$ U-40 insulin syringe to its tip at anti-mesenteric side (B. Braun Medical, S.A., Barcelona, Spain). The catheter was, then, pushed from the papilla vateri toward the biliopancreatic duct. The main hepatic canal was clamped just below the liver during the procedure, and pancreatic secretions were completely emptied. The clamp was kept in the same position during the intraductal infusion to prevent leakage into the biliary system. Then, a $3 \% 1 \mathrm{~mL} /$ $\mathrm{kg}$ taurocholic acid (Sigma, USA) solution was injected into the choledoc at a speed of $1 \mathrm{~mL} / \mathrm{min}$ under constant manual pressure using the method described by Liu et al (8). The main hepatic canal clamp was removed after the procedure and the duodenum and abdominal anterior wall were closed. All procedures were carried out under sterile conditions.

\section{Biochemical tests}

Serum amylase activity was measured with an auto-analyzer (Hitachi 917) using original kits (Boehringer, Mannheim, Germany). Measurement of Superoxide Dismutase (SOD), Malondialdehyde (MDA) and Glutathione Peroxidase (GPX) Levels in Pancreas Tissue were made in Pharmaceutical Sciences laboratories using photometric methods.

Pancreatic tissues were homogenized in $20 \mathrm{mM}$ phosphate buffer ( $\mathrm{pH}$ 7.4) containing $0.5 \mathrm{mM}$ butylated hydroxytoluene followed by a centrifugation at $4 \mathrm{C}$ at $1,500 \times \mathrm{g}$ for 15 minutes. After protein concentration measurement, equal amounts of proteins were used in triplicate to react with a chromogenic reagent
$\mathrm{N}$ - methyl-2-phenylindole to form a stable carbocyanine dye with a maximum absorption at $586 \mathrm{~nm}$. The level of MDA in sample was then calculated with the standard curve and expressed as $\mu \mathrm{mol} / \mathrm{g}$ of total protein.

The pancreatic tissue was homogenized at $4{ }^{\circ} \mathrm{C}$ in $20 \mathrm{mM}$ HEPES buffer (containing $1 \mathrm{mM}$ EGTA, $210 \mathrm{mM}$ mannitol, and $70 \mathrm{mM}$ sucrose, $\mathrm{pH}$ 7.2) and then centrifuged at $1,500 \times \mathrm{g}$ for 15 minutes. Then the radical detector (tetrazolium salt) and xanthine oxidase were added. After mixed, the sample was incubated at room temperature for $20 \mathrm{~min}$ and the absorbance was read at 450 $\mathrm{nm}$. One unit (U) of SOD was defined as the amount of enzyme needed to produce $50 \%$ dismutation of superoxide radical. The activity of SOD was expressed as U/mg of protein.

The measurement of GPX activity is based on the principle of a coupled reaction with glutathione reductase (GR). The oxidized glutathione formed after reduction of hydroperoxide by GPX is recycled to its reduced state by GR in the presence of NADPH. The oxidation of NADPH is accompanied by a decrease in absorbance at $340 \mathrm{~nm}$. One unit of GPX was defined as the amount of enzyme that catalyzed the oxidation of $1 \mathrm{nmol}$ of NADPH per minute at $25^{\circ} \mathrm{C}$. The activity of GPX was expressed as U/mg of protein.

\section{Histopathological analysis}

Collected pancreatic tissue samples were fixed in a $10 \%$ neutral buffer formaldehyde solution. In the lab, the samples were first dehydrated in ethanol (Shandon Pathcentre, UK) and, then, embedded into paraffin (Leica EG 1160, Germany). Slices of 5- $\mu \mathrm{M}$

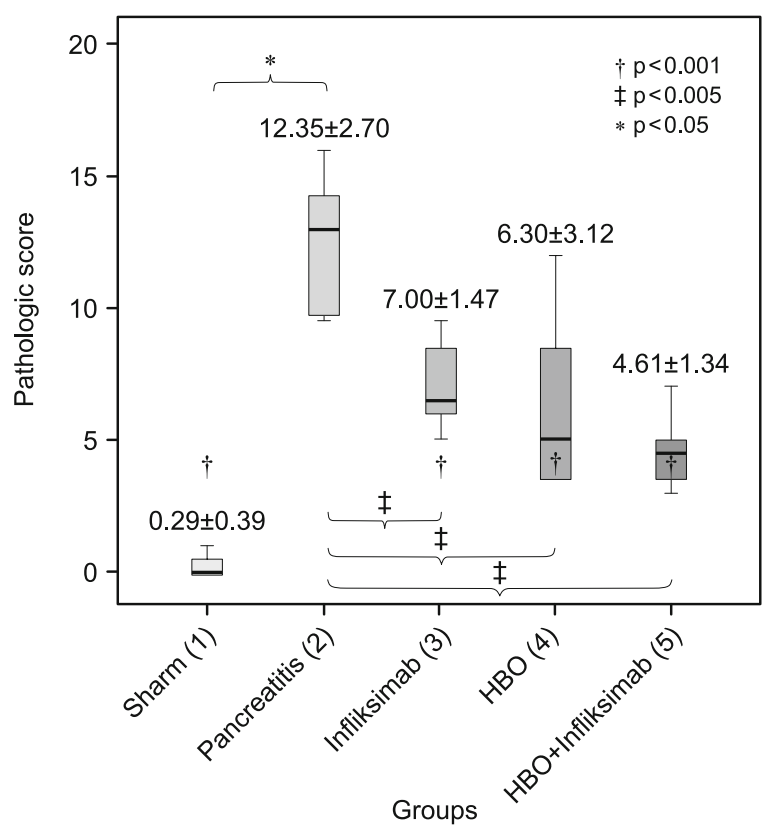

† One way ANOVA (Bonferroni corrected) (Group 1 vs 3, 4, 5) $\ddagger$ One way ANOVA (Bonferroni corrected) (Group 2 vs $3,4,5$ ) * $p<0.05$ (Group 1 vs Group 2)

Fig. 1. Comparison of histopathological scores of sham, pancreatitis and treatment groups. 


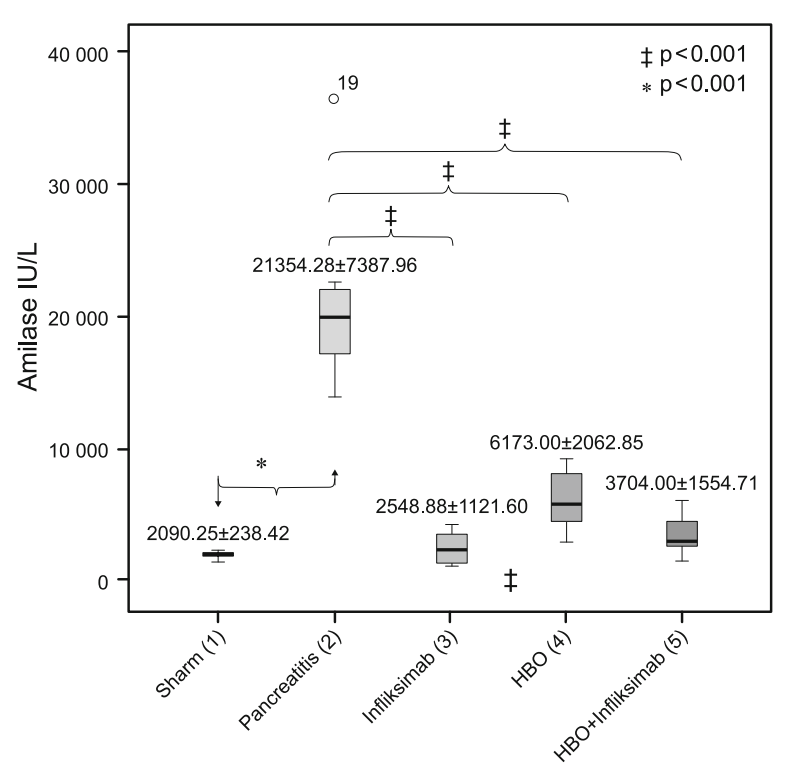

Groups

‡ One way ANOVA (Bonferroni corrected) (Group 2 vs 3, 4, 5) $* \mathrm{p}<0.05$ (Group 1 vs Group 2)

Fig. 2. Comparison of amylase levels of sham, pancreatitis and treatment groups.

thickness were obtained (Sliding Microtome, Lecia RM 2265, Germany) and the paraffin was removed using xylene. The tissue sections were stained with hematoxylin-eosin dye to be examined under light microscope (Olympus BX50, Japan). The samples were examined by two pathologists in a blinded manner, and the results were based on the scoring system defined by Schmidt et al (9) considering the degree of pancreas edema, acinar necrosis, hemorrhage and fat necrosis, inflammation, and perivascular infiltration.

\section{Statistical analysis}

Statistical analysis was performed using the SPSS version 15.0 software (SPSS Inc., Chicago, IL, USA). Continuous variables were expressed in mean \pm standard deviation (SD), while categorical variables were expressed in number and percentage. The Kolmogorov-Smirnov Goodness of Fit test was used to analyze the distribution of the data. As the data were found to be normally distributed within the groups, one-way analysis of variance (ANOVA) (Bonferroni corrected) was used to compare the continuous variables. Two-tailed $\mathrm{p}$ values of less than 0.05 were considered statistically significant.

\section{Results}

In the present study, the AP model-induced in the experimental groups was confirmed based on amylase levels and histopathology results. The mean amylase levels and histopathological scores were 2,090 IU/L and 0.29 in the sham group (Group 1), and 21,354 IU/L and 12.35 in Group 2 with induced pancreatitis $(\mathrm{p}<0.001)$ (Figs 1, 2).

When the non-treated AP group (Group 2) was compared with the treatment groups (Groups 3, 4 and 5), histopathological scores $(p<0.005)$ and amylase levels $(p<0.001)$ were found to be significantly different in all of the three groups. All of the treatments were found to be effective in AP (Figs 1, 2). The results indicated that the histopathological scores in the treatment groups improved considerably, but never decreased to sham group levels.

When the treatment groups (Groups 3, 4 and 5) were compared with each other, the histopathological scores were not found to be significantly different between Groups 3 and $4(p=0.939)$, Groups 3 and $5(\mathrm{p}=0.098)$, and Groups 4 and $5(\mathrm{p}=0.359)$. On the other hand, Group 5 had the closest histopathological score to the sham group (Figs 2, 3). When amylase levels were compared among the groups, no significant differences were observed between Groups 3 and $4(p=0.095)$, Groups 3 and $5(p=0.930)$, and Groups 4 and $5(\mathrm{p}=0.415)$.

When the levels of the parameters associated with superoxide radicals were compared among the groups (GPX, SOD, and MDA levels), the highest MDA levels were found in the AP group, indicating a significant difference compared to the sham group $(\mathrm{p}<$ 0.05 ). The MDA levels were also significantly different among all the treatment groups and non-treated AP group $(p<0.001)$ (Tab. 1). However, the SOD and GPX levels were not significantly different between Group 2 and the other treatment groups $(p=0.268$ and $\mathrm{p}=0.069$, respectively).

In total, 13 mortalities occurred in the five groups during the study: five $(41.6 \%)$ in the non-treated Group 2 , three $(25 \%)$ in Group 3, three (25\%) in Group 4 and two (16.6\%) in Group 5. The highest mortality rate was observed in the non-treated AP group, and was statistically significant $(\mathrm{p}<0.001)$ (data not shown in Tab. 1).

Tab. 1. Comparison of MDA, SOD, GPX levels of the sham group, pancreatitis group and treatment groups.

\begin{tabular}{|c|c|c|c|c|c|c|c|}
\hline & $\begin{array}{c}\text { Group 1 } \\
\text { Sham }(n=12) \\
(\text { Mean } \pm \text { SD }) \\
\end{array}$ & $\begin{array}{c}\text { Group } 2 \\
\text { Pancreatitis }(\mathrm{n}=7) \\
(\text { Mean } \pm \text { SD }) \\
\end{array}$ & $\begin{array}{c}\text { Group } 3 \\
\text { Infliximab }(\mathrm{n}=9) \\
(\text { Mean } \pm \text { SD }) \\
\end{array}$ & $\begin{array}{c}\text { Group } 4 \\
\text { HBO }(\mathrm{n}=10) \\
(\mathrm{Mean} \pm \mathrm{SD}) \\
\end{array}$ & $\begin{array}{c}\text { Group 5 } \\
\text { HBO+Infliximab }(n=7) \\
(\text { Mean } \pm \text { SD }) \\
\end{array}$ & $\begin{array}{l}p \\
\dagger\end{array}$ & $\begin{array}{l}\mathrm{p} \\
t\end{array}$ \\
\hline $\mathrm{MDA} \mu \mathrm{mol} / \mathrm{g}$ & $36.91 \pm 15.52 *$ & $109.71 \pm 6.99 *+$ & $23.77 \pm 2.53 \ddagger$ & $30.20 \pm 4.21 \ddagger$ & $25.77 \pm 3.89 \ddagger$ & $>0.05$ & $<0.001$ \\
\hline SOD U/mg & $93.58 \pm 29.04$ & $68.85 \pm 25.71$ & $85.88 \pm 13.58$ & $92.00 \pm 32.24$ & $83.77 \pm 20.52$ & $>0.05$ & $>0.05$ \\
\hline GPX U/mg & $10.88 \pm 1.88$ & $8.15 \pm 2.61$ & $9.41 \pm 1.72$ & $9.53 \pm 2.04$ & $9.05 \pm 2.41$ & $>0.05$ & $>0.05$ \\
\hline
\end{tabular}

$\uparrow$ One Way ANOVA (Bonferroni Corrected) (Group 1 vs 3, 4, 5), $¥$ (One Way ANOVA (Bonferroni Corrected) (Group 2 vs 3, 4, 5), * p $<0.05$ (Group1 vs Group 2), PAT = pathologic score, $\mathrm{MDA}=$ malondialdehyde, $\mathrm{SOD}=$ superoxide dismutase, $\mathrm{GPX}=$ glutathione peroxidase 

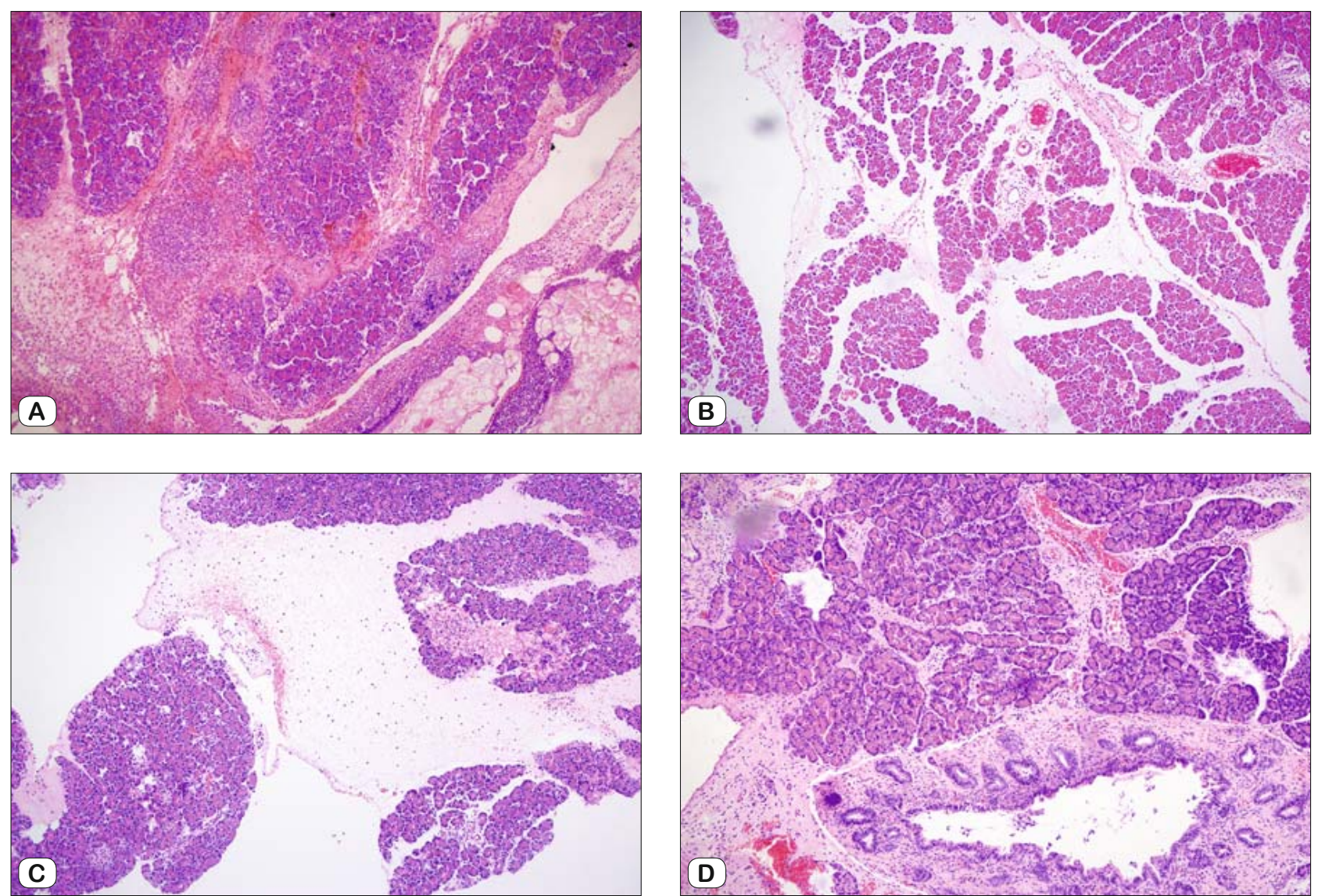

Fig. 3. Randomized histopathological examination images of the groups. Marked interlobular necrosis, inflammation and fat necrosis in the acute pancreatitis group (A), minimal edema and inflammation in the infliximab group (B), interlobular edema, focal acinar necrosis, mild interlobular inflammation in the HBO group (C), periductal minimal acute inflammation and congestion in the infliximab + HBO group (D) (Hematoxylin -Eosin, x100).

\section{Discussion}

Acute pancreatitis is a multifactorial systemic disease with several inflammatory mediators involved in its pathogenesis. Although the etiopathogenesis of the disease is well-understood, still no specific drug to neutralize the inflammatory process of pancreatitis and no single agent alone to repair the damage has been described for AP.

The histopathological grading of the pancreas has a significant role to play in the identification of the severity of AP, which can be determined based on edema, acinar necrosis, hemorrhage, fat necrosis, inflammation, and perivascular infiltration of the pancreatic tissues due to pancreatitis. The present study used the histopathological scoring system defined by Schmidt et al (8) to determine the severity of pancreatitis. With infusion of $2 \%$ taurocholate, small necrosis and lower mortality occurred compared to the infusion of $5 \%$ taurocholate, which was associated with fatal pancreatitis within hours (10). To obtain longer survival, $3 \%$ taurocholate injection was applied, and severe necrotizing acute pancreatitis occurred.

Previous studies in models of AP used serum amylase and lipase levels as parameters to demonstrate the induction of AP, as well as in its diagnosis and monitoring. Serum amylase levels start to increase within the first 3 to $6 \mathrm{~h}$ in the presence of AP, and urinary levels begin to increase within 6 to $10 \mathrm{~h}$ after the increase in serum levels (11). Serum amylase levels were found to increase significantly in the AP models induced in the present study.

Furthermore, TNF- $\alpha$ is a cytokine derived primarily from macrophages and plays a major role in the formation of many pathophysiological responses of the organism to injury or disease. Systemic symptoms in septic shock, which are associated with increased TNF- $\alpha$ levels, show a close resemblance to the symptoms of severe AP, activating leukocytes release by various mediators under the effect of TNF- $\alpha$, which is known to increase vascular permeability (12). These mediators include the platelet activating factor (PAF), nitric oxide (NO), phospholipase A2, and free oxygen radicals (13).

Tumor necrosis factor (TNF) is produced in large amounts within the pancreas, lungs, and liver during severe acute pancreatitis and is believed to mediate many of the detrimental consequences typical of this disease. In experimental models of acute pancreatitis, it has been shown that TNF- $\alpha$ gene expression begins within one hour and remains high for 1 to 3 hours, but its destruction is rapid (14). Therefore, it is aimed to prevent TNF- $\alpha$ acti- 
vity at an early stage with infliximab. In studies regarding TNF $\alpha$ inhibitors, it has been shown that maximum benefit is obtained by infliximab administration just prior to the induction of AP (4). However, it was also claimed that immediately after the induction of AP, it might be more protective (15). Thus, to prevent the activity of TNF- $\alpha$ by infliximab before induction of AP is reasonable and gives a significant biochemical and histopathologic effect.

Several studies published over the last decade demonstrated the beneficial role of TNF- $\alpha$ blockage in AP. Huges et al (14) found that the anti-TNF antibody ameliorated AP-related complications in rats. Grewal et al (12), using a polyclonal blockade of TNF, showed a statistically significant amelioration of the biochemical manifestations, although there was no significant difference in the histological grading of the pancreas. Similarly, Norman et al (16), using a soluble TNF receptor antagonist, reported a decrease in the severity and mortality of experimentally-induced pancreatitis; however, they reported no change in the degree of pancreatic vacuolization, necrosis, or inflammatory cell infiltrate. While the results of the present study are consistent with the aforementioned studies in terms of biochemical parameters, there was a statistically significant improvement in the histopathological scoring of pancreatitis. There is a number of studies that corroborate the findings of the present study, reporting improvements in both pathological parameters and amylase levels with infliximab treatment. Guice et al (17) reported an increased pancreatic edema after treatment with a TNF- $\alpha$ antagonist, and the administration of infliximab resulted in a significant improvement in biochemical and histopathological alterations in acute necrotizing pancreatitis in another study 4 . In a study by Oruc et al (18), infliximab was reported to decrease considerably, and almost significantly, pathological scores and amylase levels in both acute edematous pancreatitis and in serious necrotizing pancreatitis.

In addition, another study by Li et al (19) reported that infliximab showed beneficial effects in acute necrotizing pancreatitis by improving the barrier functions of the small intestine. In the aforementioned study, infliximab treatment improved pathological scores and amylase levels considerably, although it is obvious that infliximab treatment was not the only therapy involved in the normalization of the pathology.

Hyperbaric oxygen therapy refers to the administration of 100 $\%$ oxygen at high pressures, which promotes wound healing by amplifying the oxygen gradients along the periphery of ischemic wounds and promotes the formation of the oxygen-dependent collagen matrix formation required for angiogenesis (20-22). After preliminary studies on this topic reported favorable effects of HBO therapy on pancreatitis, the combination treatment regimens involving $\mathrm{HBO}$ became a subject of investigation. In the study by Inal et al (23), 3-amino-benzene was added to HBO therapy, and a comparative investigation of the results of the treatments was made. The results of the study showed significant decreases in the histological scores, and amylase and MDA levels. Similarly, in another experimental study performed by Uysal et al (24) on 40 rats, HBO was compared with ozone therapy, and while the effects of the treatments on pancreatitis were observed in both groups, no evaluation was made of a combined treatment. In addition, HBO therapy was not only used in experimental studies. A clinical trial performed by Lisagors et al (25) included 44 patients monitored in intensive care units for pancreatitis, of which 22 were subjected to complementary HBO therapy. Based on their results, the authors suggested that HBO therapy most probably decreased proinflammatory cytokine response and improved clinical status by increasing anti-inflammatory response. In our study, HBO used alone, and in combined therapy showed similar findings to the experimental studies. Although HBO therapy alone resulted in a greater increase in SOR and GPX activity than other treatment groups, considering pathological scores, the combination of $\mathrm{HBO}$ and TNF- $\alpha$ inhibitor therapy decreased the severity of pancreatitis more than HBO alone or TNF- $\alpha$ inhibitor alone.

The levels of oxidative stress can be identified by measuring the plasma and tissue levels of such lipid peroxidation products as GSH and MDA (26). Previous studies reported a decreased antioxidative capacity and increased lipid peroxide levels in the hepatic mitochondria of rats with an obstructed main bile duct (27). Decreased plasma levels of antioxidants and increased concentrations of MDA and similar substances enhanced SOR-induced cytotoxicity (28).

In the previous study by Tekin et al (4), the MDA levels were found to be significantly higher in the group with induced pancreatitis than in the other groups. Similarly, the MDA levels were significantly lower than those of the control group in the study by Inal et al (23). Lipid peroxidation products and anti-oxidative capacity were also evaluated in the present study, and significant decreases were observed in the MDA levels after treatment, consistent with previous studies. On the other hand, although there was no statistically significant difference in SOD and GPX activities between the AP group and treatment groups, it was observed that anti-oxidative capacities were in an increasing tendency in the treatment groups. This can be attributed to the fact that this experimental study involved a model of early-term pancreatitis, although lipid peroxidation started immediately, superoxide radicals were activated later. Accordingly, the parameters measured in the present study were not significantly different between the sham, the non-treated group, and the treatment groups.

In conclusion, the results of this study suggested that the combination of HBO therapy and TNF- $\alpha$ inhibitors showed a synergistic effect on the reduction of histopathological severity and mortality in acute pancreatitis. All the treatment modalities reduced the pathological and biochemical damage by decreasing lipid peroxidation and partly increasing the antioxidant capacity in early period, but did not show any prominent superiority to each other. Considering the fact that treatments used in this study have approved label indications for several medical conditions, we believe that they may be also appropriate for clinical use in this setting.

\section{References}

1. Buter A, Imrie CW, Carter CR, Evans S, McKay CJ. Dynamic nature of early organ dysfunction determines outcome in acute pancreatitis. Br J Surg 2002; 89: 298-302. 
417-422

2. Norman $\mathbf{J}$. The role of cytokines in the pathogenesis of acute pancreatitis. Am J Surg 1998; 175: 76-83.

3. El-Ashmawy NE, Khedr NF, El-Bahrawy HA, Hamad OB. Anti-inflammatory and Antioxidant Effects of Captopril Compared to Methylprednisolone in L-Arginine-Induced Acute Pancreatitis. Dig Dis Sci 2018; 63: 1497-1505

4. Tekin SO, Teksoz S, Terzioglu D, Arikan AE, Ozcevik H, Uslu E. Use of infliximab in treatment of acute pancreatitis. Bratisl Lek Listy 2015; 116: $167-172$.

5. Aydin S, Isik AT, Unal B, Comert B, Ozyurt M, Deveci S, Ozgur G, Cengiz O, Tasci I, Mas MR. Effects of infliximab on bacterial translocation in experimental acute necrotizing pancreatitis. Indian J Med Res 2012; 135: 656-661.

6. Isik AT, Mas MR, Comert B, Yasar M, Korkmaz A, Akay C et al. The effect of combination therapy of hyperbaric oxygen, meropenem, and selective nitric oxide synthase inhibitor in experimental acute pancreatitis. Pancreas 2004; 28: 53-57.

7. Mas N, Isik AT, Mas MR, Comert B, Tasci I, Deveci S et al. Hyperbaric oxygen-induced changes in bacterial translocation and acinar ultrastructure in rat acute necrotizing pancreatitis. J Gastroent 2005; 40: 980-986.

8. Liu Q, Djuricin G, Rossi H. The effect of lexipafant on bacterial translocation in acute necrotizing pancreatitis in rats. Am Surg 1999; 65: 611-616.

9. Schmidt J, Rattner DW, Lewandrowski K, Compton CC, Mandavilli U, Knoefel WT, Warshaw AL. A Better Model of Acute Pancreatitis for Evaluating Therapy. Ann Surg 1992; 215: 44-56.

10. Schwarz M, Thomsen J, Meyer H, Buchler MW, Beger HG. Frequency and time course of pancreatic and extrapancreatic bacterial infection in experimental acute pancreatitis in rats. Surgery 2000; 127: 427-432.

11. Jaworek J, Jachimczak B, Tomaszewska R, Konturek PC, Pawlik WW, Sendur R, Hahn EG, Stachura J, Konturek SJ. Protective Action of Lipopolysaccarides in Rat Caerulein-Induced Pancreatitis: Role Of Nitric Oxide. Digest 2000; 62: 1-13.

12. Grewal HP, Mohey el Din A, Gaber L, Kotb M, Gaber AO. Amelioration of the physiologic and biochemical changes of acute pancreatitis using an anti-TNF-alpha polyclonal antibody. Am J Surg 1994; 167: 218-219.

13. Suffys P, Beyaert R, De Valck D, Vanhae sebroeck B, Van Roy F, Fiers W. Tumour-necrosis-factor-mediated cytotoxicity is correlated with phospholipase-A2 activity, but not with arachidonic acid release per se. Eur J Biochem 1991; 195: 465-475.

14. Hughes CB, Grewal HP, Gaber LW et al. Anti-TNFalpha therapy improves survival and ameliorates the pathophysiologic sequelae in acute pancreatitis in the rat. Am J Surg 1996; 171: 274-280.

15. Norman JG, Fink GW, Messina J, Carter G, Franz MG. Timing of tumor necrosis factor antagonism is critical in determining outcome in murine lethal acute pancreatitis. Surgery 1996; 120: 515-521.
16. Norman JG, Fink GW, Franz MG. Acute pancreati tis induces intrapancreatic tumor necrosis factor gene expression. Arch Surg 1995; 130: 966-970.

17. Guice KS, Oldham KT, Remick DG, Kunkel SL, Ward PA. Antitumor necrosis factor antibody augments edema formation in caeruleininduced acute pancreatitis. J Surg Res 1991; 51: 495-499.

18. Oruc N, Ozutemiz AO, Yukselen V, Nart D, Celik HA, Yuce G, Batur Y Infliximab: a new therapeutic agent in acute pancreatitis? Pancreas 2004; 28: 1-8.

19. Li WD, Jia L, Ou Y, Jiang SM, Qiu JH, Huang YX, Du H. Infliximab: protective effect to intestinal barrier function of rat with acute necrosis pancreatitis at early stage. Pancreas 2013; 42: 366-367.

20. Gill AL, Bell CN. Hyperbaric oxygen: its uses, mechanisms of action and outcomes. QJM 2004; 97: 385-395.

21. Hunt TK. The physiology of wound healing. Ann Emerg Med 1988; 17: $1265-1273$.

22. Knighton DR, Silver IA, Hunt TK. Regulation of wound-healing angiogenesis-effect of oxygen gradients and inspired oxygen concentration. Surgery 1981; 90: 262-270.

23. Inal V, Mas MR, Isik AT, Comert B, Aydn S, Mas N, Deveci MS, Tasci I, Yamanel L, Cinar E, Unal MT. A new combination therapy in severe acute pancreatitis - hyperbaric oxygen plus 3-aminobenzamide: an experimental study. Pancreas 2015; 44: 326-330.

24. Uysal B, Yasar M, Ersoz N, Coskun O, Kilic A, Cayc T, Kurt B, Oter S, Korkmaz A, Guven A. Efficacy of hyperbaric oxygen therapy and medical ozone therapy in experimental acute necrotizing pancreatitis. Pancreas 2010; 39: 9-15.

25. Lisagors IL, Sondore A, Pupelis G, Oshs P, Iaunalksne I, Pavars M, Arons M. Impact of hyperbaric oxygen therapy on the clinical course of acute pancreatitis and systemic inflammation response syndrome Anesteziol Reanimatol 2008; 4: 34-38.

26. Galley H, Davies M, Webster N. Xanthine Oxidase Activity and Free Radical Generation in Patients with Sepsis Syndrome. Crit Care Med 1996; 24: 1649-1653.

27. Sokol RJ, McKim Jr JM, Goff Colby M, Ruyle SZ, Devereaux MW, Han D et al. Vitamin E reduces oxidant injury to mitochondria and the hepatotoxicity of taurochenodeoxycholic acid in the rat. Gastroent 1998; 114: 164-174.

28. Tsai LY, Lee KT, Liu TZ. Evidence for accelerated generation of hydroxyl radicals in experimental obstructive jaundice of rats. Free Radic Biol Med 1998; 24: 732-737.

Received March 10, 2019. Accepted April 16, 2019. 